\title{
Registration of Longitudinal Image Sequences with Implicit Template and Spatial-Temporal Heuristics
}

\author{
Guorong $\mathrm{Wu}^{1}$, Qian Wang ${ }^{1,2}$, Hongjun $\mathrm{Jia}^{1}$, and Dinggang Shen ${ }^{1}$ \\ ${ }^{1}$ Department of Radiology and BRIC, University of North Carolina at Chapel Hill \\ \{grwu, jiahj, dgshen\} @med.unc. edu \\ ${ }^{2}$ Department of Computer Science, University of North Carolina at Chapel Hill \\ qianwang@cs. unc .edu
}

\begin{abstract}
Accurate measurement of longitudinal changes of anatomical structure is important and challenging in many clinical studies. Also, for identification of disease-affected regions due to the brain disease, it is extremely necessary to register a population data to the common space simultaneously. In this paper, we propose a new method for simultaneous longitudinal and groupwise registration of a set of longitudinal data acquired from multiple subjects. Our goal is to 1) consistently measure the longitudinal changes from a sequence of longitudinal data acquired from the same subject; and 2) jointly align all image data (acquired from all time points of all subjects) to a hidden common space. To achieve these two goals, we first introduce a set of temporal fiber bundles to explore the spatial-temporal behavior of anatomical changes in each longitudinal data of the same subject. Then, a probabilistic model is built upon the hidden state of spatial smoothness and temporal continuity on the fibers. Finally, the transformation fields that connect each time-point image of each subject to the common space are simultaneously estimated by the expectation maximization (EM) approach, via the maximum a posterior (MAP) estimation of probabilistic models. Promising results are obtained to quantitatively measure the longitudinal changes of hippocampus volume, indicating better performance of our method than the conventional pairwise methods.
\end{abstract}

\section{Introduction}

Longitudinal study of human brains is important to reveal subtle structural and functional changes due to the brain disease $[1,2]$. Although many image registration algorithms have been developed, most of them, regardless of feature-based or intensity-based, register the serial images independently when applied to longitudinal data. However, the independent registration implies the independent measurement of brain changes in serial images, and could lead to inconsistent longitudinal measurement especially for the small structures with tiny annual changes (e.g., atrophy in hippocampus due to aging [3]).

Several methods have been reported to measure the temporal anatomical changes. Shen and Davatzikos [3] proposed the 4D-HAMMER registration to cater for the longitudinal application. However, one limitation of their method is to build a 4D template by repeating one specific 3D image as templates for different time-points, which may introduce bias in longitudinal data analysis. Recently, Durrleman et al. [4] presented a spatial-temporal atlas estimation method to analyze the variability of the 
longitudinal shapes. This method uses the regression model to infer the shape evolution, and then jointly estimates the deformations as well as the dynamics of temporal changes. However, this method also needs to explicitly specify a template in advance.

In this paper, we propose a novel longitudinal registration method for image sequences acquired at multiple time-points of multiple subjects. Our method integrates both advantages of groupwise registration and longitudinal registration, thus achieving the spatial-temporal consistency for the registration results. Specifically, the transformation fields of all images, regardless of subjects and acquisition times, are first simultaneously estimated towards a hidden common space. Second, the spatialtemporal consistency of deformations within serial images of each subject is regulated by a set of temporal fiber bundles [5] to convey the spatial dependence and temporal continuity. Finally, these two steps are naturally integrated by a probabilistic model on fiber bundles and further solved via an EM framework. Promising results of the proposed method are obtained on both simulated and real longitudinal brain data with hippocampus atrophy, indicating better performance of our method than the conventional pairwise registration methods.

\section{Methods}

Given a set of longitudinal image sequences, $\boldsymbol{I}=\left\{I_{s, t} \mid s=1, \ldots, N, t=1, \ldots, T_{s}\right\}$ where $I_{s, t}$ represents the image acquired from subject $s$ at time point $t$, our first goal is to align them to a common space following the transformation field $f_{s, t}$ defined from the common space to the individual image space of $I_{s, t}$. We define the mean shape of all aligned brain boundaries as $Z=\left\{z_{j} \mid j=1, \ldots, K\right\}$, where $z_{j}$ denotes the position of the $j$-th point of the mean shape in the common space. Then, for each $z_{j}$, its transformed positions in the space of subject $s$, denoted as $\Phi_{s, j}=\left\{f_{s, t}\left(z_{j}\right) \mid t=\right.$ $\left.1, \ldots, T_{s}\right\}$, can be regarded as a temporal fiber within the subject $s$, which facilitates the regulation of the temporal continuity for the longitudinal data. Fig. 1 illustrates the main idea of our registration method. As shown in Fig. 1(a), all corresponding points in the longitudinal sequences of different subjects (i.e., colored dots) are linked to the common space (i.e., red star) based on their respective deformation fields $f_{s, t}$, and also their longitudinal changes within each subject (i.e., dash curves) are captured by the temporal fiber bundles $\left\{\Phi_{s, j}\right\}$. Fig. 1(b) shows more details on registering a set of longitudinal data. Specifically, the mean shape $Z$ is given in the top, with all its instances $f_{s, t}(Z)$ shown in the middle, where all images of each longitudinal data are connected by the embedding temporal fibers $\left\{\Phi_{s, j}\right\}$. To establish the point correspondences w.r.t. the common space, only the most distinctive voxels, called as driving voxels $X_{s, t}$, are used for to drive the transformation of each image $I_{s, t}$. Examples of driving voxels are given in the bottom of Fig. 1(b) as red points overlaid on brain slices. Note that each transformation field $f_{s, t}$ is steered by the correspondences determined between $X_{s, t}$ and $f_{s, t}(Z)$, with the estimated transformation fields of the same subject regularized along each temporal fiber $\Phi_{s, j}$, to achieve the temporal continuity. The hidden states of spatial-temporal characteristics on fiber bundles will be described by a probabilistic model introduced in Section 2.1. We will present a solution to this probabilistic model in Section 2.2, where the estimation of transformation fields $f_{s, t}$ is regarded as the MAP estimation of model parameters. 

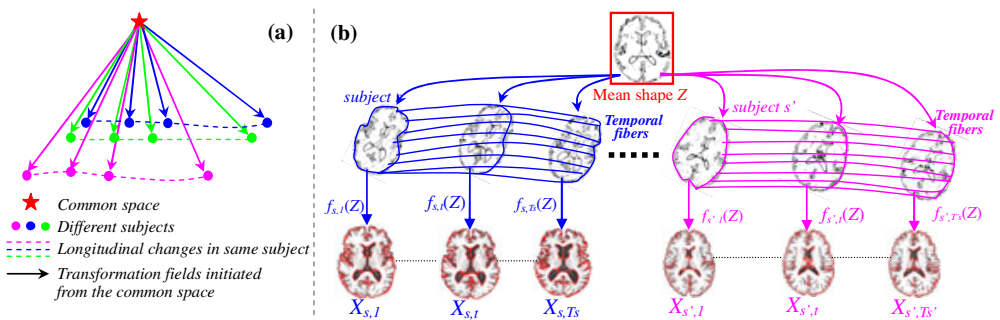

Fig. 1. The schematic illustration of the proposed method for simultaneous longitudinal and groupwise registration. In (a), not only all subjects are jointly warped onto the common space, but also the longitudinal changes are consistently measured by fibers in longitudinal data of each subject. In (b), an overview of our method is provided. For each image, the driving voxels $X_{s, t}$ (shown in red in the bottom) will be used to steer the estimation of transformation field $f_{s, t}$, by establishing their correspondences with the mean shape in the common space (as shown in the top). Meanwhile, the temporal consistency within each longitudinal data can be regulated along the temporal fiber bundles, as displayed by lines in the middle of (b).

\subsection{Probabilistic Model for Registration of Longitudinal Data Sets}

Our registration aims to simultaneously minimize the variability of serial images of all subjects, while enforcing the temporal continuity within the longitudinal data of each subject. In general, the transformation field $f_{s, t}$ (defined in the common space) is used to pull each data $I_{s, t}$ from its own space to the common space. The bending energy is used as a smoothness constraint to regularize each $f_{s, t}$, since thin-plate splines (TPS) [6] is efficient to give the optimal $f_{s, t}$ with minimal bending energy. In the following, we will use the operator $L_{S}\left(f_{s, t}\right)$ to denote the bending energy of each transformation field $f_{s, t}$ in the spatial domain.

Gaussian Mixture Model on the Driving Voxels: Attribute vector has been widely used as a morphological signature to guide image registration. In this paper, we follow the method in [7] to calculate an attribute vector $\vec{a}(x)$ at each location $x \in \Re^{3}$ in each subject image $I_{s, t}$. Based on attribute vectors, the set of driving voxels in $I_{s, t}$, denoted as $X_{s, t}=\left\{X_{s, t}^{i} \mid i=1, \ldots, M_{s, t}\right\}$ where $M_{s, t}$ is the total number of the driving voxels in $I_{s, t}$, can be selected for steering the registration [7]. Here, we regard each $X_{s, t}^{i}$ as the random variable drawn from a Gaussian mixture model (GMM) whose centers are the deformed point set $f_{s, t}(Z)$. Accordingly, we introduce a hidden variable $m_{s, t}^{i} \in\{1, \ldots, K\}$ (where $K$ is the number of temporal fibers) to indicate the spatial assignment of a specific GMM center in generating $X_{s, t}^{i}$. The complete density function of $X_{s, t}^{i}$ can be given by:

$$
P\left(X_{s, t}^{i} \mid m_{s, t}^{i}=j, f_{s, t}, Z_{j}\right)=N\left(X_{s, t}^{i} ; f_{s, t}\left(Z_{j}\right), \Sigma_{s, t}^{j}\right) \propto \exp \left\{-\frac{\left\|X_{s, t}^{i}-f_{s, t}\left(Z_{j}\right)\right\|^{2}}{\sigma^{2}}\right\},
$$

where $\Sigma_{s, t}^{j}$ is the covariance matrix of the normal distribution $N(X ; \mu, \Sigma)$. For sake of simplicity, the isotropic covariance matrix $\Sigma_{s, t}^{j}=\sigma^{2} \cdot I_{K \times K}$ is assumed.

Given the spatial assignment $m_{s, t}^{i}$, the attribute vector on $X_{s, t}^{i}$, i.e., $\vec{a}\left(X_{s, t}^{i}\right)$, can be also viewed as a random variable generated from another GMM whose centers are the 
respective attribute vectors on all other images, denoted as $\vec{a}\left(f_{s^{\prime}, t^{\prime}}\left(Z_{j}\right)\right)\left(s^{\prime} \neq s, t^{\prime} \neq\right.$ $t$ ). We introduce a subject assignment $n_{s, t}^{i} \in\left\{\left(s^{\prime}, t^{\prime}\right)\right\}$ to denote the index of image data for reference. Similarly, the conditional density function of $\vec{a}\left(X_{s, t}^{i}\right)$ is defined as:

$$
\begin{gathered}
P\left(\vec{a}\left(X_{s, t}^{i}\right) \mid X_{s, t}^{i}, m_{s, t}^{i}=j, n_{s, t}^{i}=\left(s^{\prime}, t^{\prime}\right), f_{s, t} f_{\left.s^{\prime}, t^{\prime}, Z_{j}\right)}\right. \\
=N\left(\vec{a}\left(X_{s, t}^{i}\right) ; \vec{a}\left(f_{s^{\prime}, t^{\prime}}\left(Z_{j}\right)\right), \Sigma_{s, t}^{j}\right) \propto \exp \left\{-\frac{\left\|\vec{a}\left(X_{s, t}^{i}\right)-\vec{a}\left(f_{s^{\prime}, t^{\prime}}\left(Z_{j}\right)\right)\right\|^{2}}{\tau^{2}}\right\},
\end{gathered}
$$

where we assume again the isotropic covariance matrix.

By denoting the occupancy probabilities of $m_{s, t}^{i}$ and $n_{s, t}^{i}$ as $P\left(m_{s, t}^{i}=j\right)=\pi_{s, t}^{i}$ and $P\left(n_{s, t}^{i}=\left(s^{\prime}, t^{\prime}\right)\right)=\omega_{s, t}^{i}$, respectively, the posterior probability of $\left(X_{s, t}^{i}, \vec{a}\left(X_{s, t}^{i}\right)\right)$ given the transformation field $f_{s, t}$ and the mean shape $Z$ can be given as:

$$
\begin{gathered}
P\left(X_{s, t}^{i}, \vec{a}\left(X_{s, t}^{i}\right) \mid f_{s, t}, Z_{j}\right)=\sum_{s=1}^{N} \sum_{j=1}^{K} \pi_{s, t}^{i} \cdot \omega_{s, t}^{i} \cdot \exp \left(-\xi_{s, t}^{i}\left(\pi_{s, t}^{i}, \omega_{s, t}^{i}\right)\right) \\
\xi_{s, t}^{i}\left(\pi_{s, t}^{i}, \omega_{s, t}^{i}\right)=\frac{\left\|X_{s, t}^{i}-f_{s, t}\left(Z_{j}\right)\right\|^{2}}{\sigma^{2}}+\frac{\left\|\vec{a}\left(X_{s, t}^{i}\right)-\vec{a}\left(f_{s^{\prime}, t^{\prime}}\left(Z_{j}\right)\right)\right\|^{2}}{\tau^{2}} .
\end{gathered}
$$

Spatial-Temporal Heuristics on Fiber Bundles: For each subject $\boldsymbol{s}$, the temporal fiber bundles capture the longitudinal consistency of the estimated deformations, as shown in Fig. 1. In other words, we can take advantage of the temporal fiber bundles, designated by $\left(\boldsymbol{f}_{s, \boldsymbol{t}}, \boldsymbol{t}=\mathbf{1}, \ldots, \boldsymbol{T}_{s}\right)$ from the common space, to regulate the temporal smoothness from $\boldsymbol{f}_{\boldsymbol{s}, \mathbf{1}}\left(\boldsymbol{Z}_{\boldsymbol{j}}\right)$ to $\boldsymbol{f}_{\boldsymbol{s}, \boldsymbol{T}_{s}}\left(\boldsymbol{Z}_{\boldsymbol{j}}\right)$ along the $\boldsymbol{j}$-th fiber. A traditional kernel-based approach can be used here to assure the temporal continuity. In particular, the position of $\boldsymbol{f}_{\boldsymbol{s}, \boldsymbol{t}}\left(\boldsymbol{Z}_{\boldsymbol{j}}\right)$ after kernel smoothing is defined by:

$$
\hat{f}_{s, t}\left(Z_{j}\right)=\sum_{\tau=1}^{T_{s}} G\left(\frac{\tau-t}{w}\right) \cdot f_{s, \tau}\left(Z_{j}\right) / \sum_{\tau=1}^{T_{s}} G\left(\frac{\tau-t}{w}\right),
$$

where $G$ is a Gaussian kernel and $w$ the kernel width. The principle of Gaussian kernel smoothing leads to the minimization of the deviation between $f_{s, t}\left(Z_{j}\right)$ and $\hat{f}_{s, t}\left(Z_{j}\right)$. We use the operator $L_{T}\left(f_{s, t}\left(Z_{j}\right)\right)=\left\|\hat{f}_{s, t}\left(Z_{j}\right)-f_{s, t}\left(Z_{j}\right)\right\|^{2}$ to measure the residual energy after performing temporal smoothing on each position of a fiber, which accounts for the smoothness in temporal domain. It is worth noting that more sophisticated kernel-based regression methods are also applicable here.

Unified Probabilistic Model for Registration of Longitudinal Data Sets: Considering $h=\left(\left\{Z_{j}\right\},\left\{f_{s, t}\right\},\left\{m_{s, t}^{i}\right\},\left\{n_{s, t}^{i}\right\}\right)$ as the model parameters and $v=\left(\left\{X_{s, t}^{i}\right\},\left\{\vec{a}\left(X_{s, t}^{i}\right)\right\}\right)$ as the observation, the joint probability $P(h, v)$ is given by:

$$
\begin{aligned}
& P(h, v) \propto\left[\prod_{j=1}^{K} P\left(Z_{j}\right)\right] \cdot\left[\prod_{s=1}^{N} P\left(f_{s, t}\right) \cdot \prod_{j=1}^{K} \prod_{t=1}^{T_{s}} P\left(f_{s, t}\left(Z_{j}\right)\right)\right] \cdot\left[\prod_{i=1}^{M_{s, t}} P\left(X_{s, t}^{i}, \vec{a}\left(X_{s, t}^{i}\right) \mid f_{s, t}, Z_{j}\right)\right], \\
& P\left(f_{s, t}\right)=e^{-\frac{\lambda_{1}}{2}\left\|L_{S}\left(f_{s, t}\right)\right\|^{2}}, P\left(f_{s, t}\left(Z_{j}\right)\right)=e^{-\frac{\lambda_{2}}{2}\left\|L_{T}\left(f_{s, t}\left(Z_{j}\right)\right)\right\|^{2}},
\end{aligned}
$$

where $P\left(Z_{j}\right)=\frac{1}{K}$ due to no prior knowledge on $Z_{j}$.

Hereafter, our registration problem turns to infer the model parameters $h$ that best interpret the observation $v$, i.e., $\hat{h}=\arg \max _{h} P(h, v)$. 


\subsection{Solutions to Our Longitudinal Registration}

Finding the best model parameters $\hat{h}$ by MAP estimation on $P(h, v)$ is intractable since many parameters $h$ might explain the observation $v$ very well. "Free energy with annealing" [8] is one of the variational Bayes inference approach to solve this problem by minimizing the distribution distance between $p(h \mid v)$ and $p(h, v)$, which bounds for maximizing the likelihood of the observation $P(v)$. However, here the posteriori distributions of some variables $\left(f_{s, t}, \hat{f}_{s, t}\right.$, and $\left.Z\right)$ are hard to compute. Instead, we perform the point estimation on $f_{s, t}, \hat{f}_{s, t}$, and $Z$ by maximizing their expectation w.r.t. the maintained posteriori distributions of $\left\{m_{s, t}^{i}\right\}$ and $\left\{n_{s, t}^{i}\right\}$, which falls to the EM principle. By omitting some constant terms and taking logarithm to Eq. 5, we obtain the free energy for our registration algorithm as:

$$
\begin{gathered}
E(\pi, \omega, f, \hat{f}, Z) \propto \sum_{s=1}^{N} \sum_{t=1}^{T_{s}} \sum_{j=1}^{K} \sum_{i=1}^{M_{s, t}}\left[\pi_{s, t}^{i} \cdot \omega_{s, t}^{i} \cdot \xi_{s, t}^{i}\left(\pi_{s, t}^{i}, \omega_{s, t}^{i}\right)+\left(r_{1} \cdot \pi_{s, t}^{i} \cdot \log \left(\pi_{s, t}^{i}\right)+r_{2} \cdot \omega_{s, t}^{i} \cdot \log \left(\omega_{s, t}^{i}\right)\right)\right] \\
+\sum_{s=1}^{N} \frac{\lambda_{1}}{2} L_{s}\left(f_{s, t}\right)+\frac{\lambda_{2}}{2} \sum_{s=1}^{N} \sum_{j=1}^{K} \sum_{t=1}^{T_{s}} L_{T}\left(f_{s, t}\left(Z_{j}\right)\right),
\end{gathered}
$$

where $r_{1}$ and $r_{2}$ are the "temperatures" for spatial assignment and subject assignment, respectively, in annealing scenario. It is worth noting that $r_{1}$ and $r_{2}$ will gradually decrease to encourage the assignment changing from fuzzy to strictly binary.

The spatial assignment $\pi_{s, t}^{i}$ and subject assignment $\omega_{s, t}^{i}$ can be obtained by minimizing $E$ w.r.t. $\pi_{s, t}^{i}$ and $\omega_{s, t}^{i}$, respectively:

$$
\begin{array}{ll}
\hat{\pi}_{s, t}^{i}=\exp \left\{-\frac{\omega_{s, t}^{i}}{r_{1}} \cdot\left(\xi_{s, t}^{i}\left(\pi_{s, t}^{i}, \omega_{s, t}^{i}\right)\right)\right\}, & \hat{\pi}_{s, t}^{i} \leftarrow \hat{\pi}_{s, t}^{i} / \sum_{j=1}^{K} \hat{\pi}_{s, t}^{i} \\
\widehat{\omega}_{s, t}^{i}=\exp \left\{-\frac{\pi_{s, t}^{i}}{r_{2}} \cdot\left(\xi_{s, t}^{i}\left(\pi_{s, t}^{i}, \omega_{s, t}^{i}\right)\right)\right\}, & \widehat{\omega}_{s, t}^{i} \leftarrow \widehat{\omega}_{s, t}^{i} / \sum_{s=1}^{N} \widehat{\omega}_{s, t}^{i}
\end{array}
$$

Next, Eq. 6 can be optimized by decoupling it into two sub-problems, i.e., (SP $\mathbf{1})$ tracking the longitudinal changes along the fiber bundles; and $\left(\mathbf{S P}_{\mathbf{2}}\right)$ simultaneously estimating the transformation fields $f_{s, t}$ and updating the mean shape $Z$.

SP $_{1}$ : Longitudinal Tracking along Fiber Bundles: Once the mean shape $Z$ and transformation $f_{s, t}$ of each image have been determined, temporal continuity is preserved by tracking the composite variables from $f_{s, 1}\left(Z_{j}\right)$ to $f_{s, T_{S}}\left(Z_{j}\right)$ in each temporal fiber $Z_{j}$. Here, kernel-based smoothing is performed along each fiber of individual subject, to enforce the temporal continuity by Eq. 4 and minimizes the energy function $E$ in Eq. 6 w.r.t. $f_{s, t}\left(Z_{j}\right)$.

$\mathbf{S P}_{2}$ : Groupwise Registration: This step aims to refine $f_{s, t}$ and $Z$ by minimizing the energy function $E$ with fixed $\pi, \omega$, and $\hat{f}_{s, t}$. This can be considered as a groupwise registration problem, which is solved in three iterative steps. First, the transformation $f_{s, t}\left(Z_{j}\right)$ w.r.t. to all driving voxels $X_{x, t}^{i}$ in $I_{s, t}$ is updated as a weighted mean location according to the observations of $\pi_{s, t}^{i}$ and $\omega_{s, t}^{i}$, i.e., $f_{s, t}\left(Z_{j}\right)=\sum_{i=1}^{M_{s, t}} \pi_{s, t}^{i} \cdot \omega_{s, t}^{i} \cdot X_{s, t}^{i}$. Second, by considering $\left\{Z_{j}\right\}$ as the source point set and $\left\{f_{s, t}\left(Z_{j}\right)\right\}$ as the target point set, TPS interpolates the dense transformation field $f_{s, t}$ for each $I_{s, t}$. Finally, by 
minimizing $E$ with respect to $Z_{j}$, the mean shape $Z$ is updated as $\hat{Z}_{j}=\sum_{s=1}^{N} \sum_{t=1}^{T_{s}} \widehat{\omega}_{s, t}^{i} \sum_{i=1}^{M_{s, t}} \hat{\pi}_{s, t}^{i} f_{s, t}^{-1}\left(X_{s, t}^{i}\right)$, where $f_{s, t}^{-1}$ is the inverse of the transformation field $f_{s, t}$.

\section{Experiments}

The ultimate goal of our longitudinal registration algorithm is to consistently measure the subtle anatomical changes to facilitate the diagnosis (or early prediction) of diseases, i.e., brain dementia. To evaluate the performance of our method, two sets of data (one with simulated atrophy in hippocampus, and another with manually labeled hippocampi in year 1 and year 5 of 9 elder brains) are used for demonstration in this section. For comparison, we have also used a 3D pairwise registration algorithm [9] to independently measure the longitudinal changes of hippocampi for these data.

Experiments on Simulated Atrophy in Hippocampus: Three subjects with manually labeled hippocampus regions at year 1 are used as the baseline data $(t=1)$ to simulate atrophy on their hippocampi. Then, we yield the next time-point image $(t=2)$ by simulating $\sim 5 \%$ hippocampal shrinkage. Repeating this procedure, we finally obtain 3 sets of longitudinal data with hippocampal volume shrinking at an annual rate of $\sim 5 \%$ in the five years. Considering the numerical error in simulation, the total simulated atrophy is $15.86 \%$ within the five years.

Our algorithm can simultaneously estimate the transformation fields of these $5 \times 3$ images from their own spaces to the hidden common space, and keep spatial-temporal continuity on the estimated transformation fields for each subject. Fig. 2(a) shows the curves of the estimated hippocampus volume loss by a 3D pairwise registration method and our proposed method in blue and red curves, respectively. It is obvious that our method outperforms the 3D pairwise registration method, with more consistent measurement of longitudinal changes. The averaged hippocampus volume loss estimated by the two methods is also displayed in Fig. 2(b). The final estimated hippocampus volume loss is $12.8 \%$ by the 3D pairwise registration method and $15.73 \%$ by our method, which shows the capability of our method in more accurate measurement of anatomical changes.

Experiments on Real Elderly Brains: Nine elderly subjects, each with annual MR scans in 5 years, are evaluated in this experiment. For comparison, we independently warp these $9 \times 5$ subjects to a selected template by the $3 \mathrm{D}$ pairwise registration method. The registration results of all five time-points of the two subjects are shown in the top two rows of Fig. 3(a). We also perform our longitudinal registration method to simultaneously align these 45 subjects to the hidden common space without specifying the template. The registration results of the same two subjects across 5 years are displayed in the top two rows of Fig. 3(b). It can be observed that our method achieves more consistent alignment in global brain shapes. We further enlarge the right pre-/post-central gyrus area, as shown in two bottom rows. As indicated by the pink crosses, the registration results along the time axis are not continuous for the $3 \mathrm{D}$ method, while the longitudinal registration results of our method are more consistent, due to the introduction of the spatial-temporal heuristics. Noting that the percentage 
of hippocampal shrinkage detected from year 1 to year 5 is $3.84 \%$ by $3 \mathrm{D}$ method and $5.38 \%$ by our method, compared to $5.65 \%$ by two experienced raters.

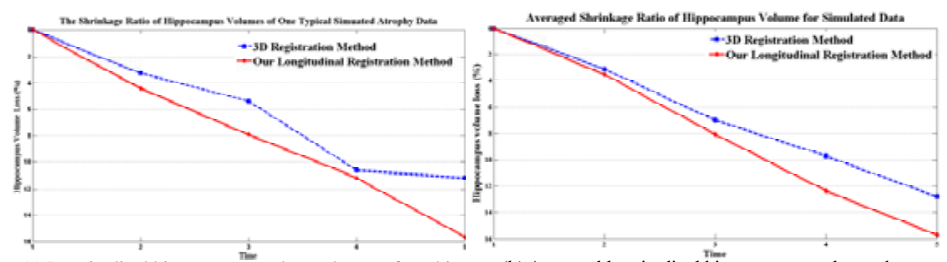

(a) Longitudinal hippocampus volume change of a subject

(b) Averaged longitudinal hippocampus volume change

Fig. 2. Experimental results on measuring simulated hippocampus volume loss in the 5 years. (a) shows the hippocampus volume loss for a typical subject in the 5 years and (b) shows the averaged hippocampus volume change in the 5 years. The total simulated atrophy in the 5 years is $15.86 \%$. The estimated atrophy is $15.73 \%$ by our method and $12.8 \%$ by the 3D method, indicating more accurate results achieved by our method.

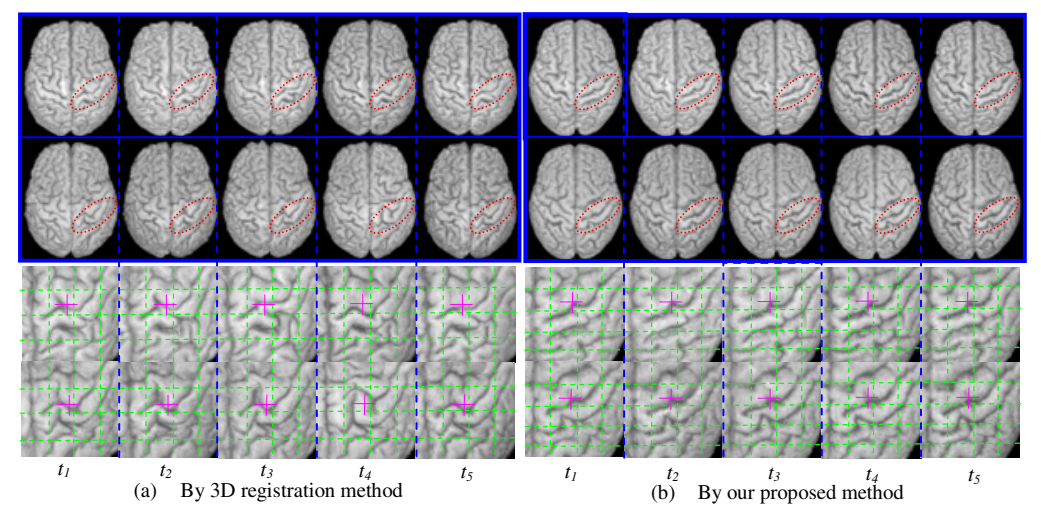

Fig. 3. Registration results on two typical subjects (with 5 scans) by 3D pairwise registration method (a) and our proposed method (b). The top two rows show the 3D rendering of two aligned subjects in 5 time points. Visually, our method achieves more consistent results in the global brain shapes (particularly for the regions indicated by the red ellipses). The bottom two rows show an enlarged region at right pre-/post-central gyri area. As indicated by pink crosses, our method outperforms the pairwise registration method in temporal continuity.

The registration performance can be further evaluated by computing the overlap ratio on brain tissues between each aligned image and the reference image. In our algorithm, since no explicit template is used, the reference image is voted from all aligned images by setting the tissue at each voxel as the majority of tissue labels from all aligned images. First, by considering all 45 aligned images together, the average overlap ratio of white matter (WM), gray matter (GM), ventricle (VN) are $67.2 \%$, $59.2 \%, 79.0 \%$ by the 3D method, while $72.6 \%, 63.9 \%$, and $82.1 \%$ by our method. Second, by considering all 5 aligned scans of each subject as a separate group, the mean and standard deviation of overlap ratios from 9 subjects are $(79.7 \% \pm$ $2.5 \%),(71.3 \% \pm 2.4 \%),(86.3 \% \pm 1.7 \%)$ by the $3 \mathrm{D}$ method on WM, GM, and VN, 
respectively, compared to $(82.1 \% \pm 1.4 \%),(74.5 \% \pm 2.1 \%),(86.6 \% \pm 1.3 \%)$ by our method. Obviously, these two sets of results consistently demonstrate that our method outperforms the 3D method in accurately aligning all 45 images to the common space (first experiment), as well as better preserving the temporal continuity in each subject (second experiment).

\section{Conclusion}

We have presented a novel method for longitudinal registration of serial images, by taking advantages of both spatial-temporal heuristics and implicit template in registering a group of longitudinal data. Specifically, the spatial-temporal continuity is achieved by enforcing smoothness constraint along fiber bundles in each subject. Further, we simultaneously estimate all transformation fields which link all images to the hidden common space without specifying any template, thus avoid introduction of any bias to subsequent data analysis.

In future, we will investigate more powerful kernel regression methods to regulate the temporal smoothness along the temporal fiber bundles. Also, we will test our longitudinal registration method in clinical applications, e.g., measuring longitudinal brains change in MCI study.

\section{References}

1. Cardenas, V.A., Du, A.T., Hardin, D., Ezekiel, F., Weber, P., Jagust, W.J., Chui, H.C., Schuff, N., Weiner, M.W.: Comparison of methods for measuring longitudinal brain change in cognitive impairment and dementia. Neurobiology of Aging. 24(4), 537-544 (2003)

2. Toga, A.W., Thompson, P.M.: Temporal dynamics of brain anatomy. Annu. Rev. Biomed. Eng. 5, 119-145 (2003)

3. Shen, D., Davatzikos, C.: Measuring Temporal Morphological Changes Robustly in Brain MR Images Via 4-Dimensional Template Warping. NeuroImage 21(4), 1508-1517 (2004)

4. Durrleman, S., Pennec, X., Trouve, A., Gerig, G., Ayache, N.: Spatiotemporal Atlas Estimation for Developmental Delay Detection in Longitudinal Datasets. In: Yang, G., Rueckert, D., Noble, A., Taylor, C. (eds.) MICCAI 2009, Part I. LNCS, vol. 5761, pp. 297-304. Springer, Heidelberg (2009)

5. Di, H., Tao, L., Xu, G.: A Mixture of Transformed Hidden Markov Models for Elastic Motion Estimation. IEEE Transactions on Pattern Analysis and Machine Intelligence 31(10), 1817-1830 (2009)

6. Bookstein, F.L.: Principal Warps: Thin-Plate Splines and the Decomposition of Deformations. IEEE Transactions on Pattern Analysis and Machine Intelligence 11(6), 567-585 (1989)

7. Shen, D., Davatzikos, C.: HAMMER: Hierarchical attribute matching mechanism for elastic registration. IEEE Transactions on Medical Imaging 21(11), 1421-1439 (2002)

8. Frey, B.J., Jojic, N.: A Comparison of Algorithms for Inference and Learning in Probabilistic Graphical Models. IEEE Transactions on Pattern Analysis and Machine Intelligence 27(9), 1392-1416 (2005)

9. Wu, G., Yap, P.T., Kim, M., Shen, D.: TPS-HAMMER: Improving HAMMER registration algorithm by soft correspondence matching and thin-plate splines based deformation interpolation. NeuroImage 49(3), 2225-2233 (2010) 